\title{
Effectiveness of menthol and folium menthae piperitae against Acinetobacter baumannii
}

\author{
Ertugrul Guclu ${ }^{1 \star}$, Hayriye Genc ${ }^{2}$, Mustafa Zengin ${ }^{2}$ and Oguz Karabay ${ }^{1}$ \\ ${ }^{1}$ Department of Infectious Diseases and Clinical Microbiology, Ministry of Health Sakarya University Training and \\ Research Hospital, Sakarya, Turkey. \\ ${ }^{2}$ Department of Chemistry, Sakarya University Art and Science Faculty, Sakarya, Turkey.
}

Accepted 22 May, 2013

\begin{abstract}
Acinetobacter baumannii is a common Gram negative nosocomial pathogen. Although new agents have been developed against Gram positive microorganisms, there are few new antibacterial agents against Gram negative bacteria. In this environment, herbal medicine has become a flicker of hope. The purpose of this study was to investigate the antibacterial activity of menthol and folium menthae piperitae extract either alone or as a mixture against $A$. baumannii. A total of 20 multidrug resistant $A$. baumannii strains that had been isolated from hospitalized patients as nosocomial pathogens was used. The antibacterial activity of test materials was assessed by both quantitative suspension test and an agar well diffusion test. Menthol was found to be effective against $\boldsymbol{A}$. baumannii. The mixture of menthol and folium menthae piperitae extract was also found to have antibacterial activity. However, folium menthae piperitae extract alone had no antibacterial activity against $A$. baumannii with a contact time of one minute, whereas it was effective with contact times greater than one minute. We conclude that topical form of menthol can be used for skin and soft tissue infections, burn wounds, diabetic foot infections and decubitus wound infections caused by $A$. baumannii.
\end{abstract}

Key words: Menthol, menthae piperitae, Acinetobacter baumannii.

\section{INTRODUCTION}

Recently, antimicrobial resistant Gram-negative bacilli have become a common nosocomial pathogen in health care settings (Kunz et al., 2010). Multidrug resistant (MDR) Pseudomonas aeruginosa, extended spectrum beta-lactamase (ESBL) producing Enterobacteriaceae and carbapenem-resistant Acinetobacter baumanii are the most common Gram-negative nosocomial microorganisms isolated from patients in the intensive care unit. The continuing increase in antimicrobial resistance throughout the world remains a concern (Kunz et al, 2010; Rosenthal et al.,2010).

Acinetobacter is a group of bacteria commonly found in soil and water. While there are many types or "species" of Acinetobacter and all can cause human disease, $A$. baumannii is the most frequently reported type (CDC, 2010). A. baumannii is a nonfermentative, gram-negative, nonmotile, and oxidase-negative cocobacillary rod that grows at $20-30^{\circ} \mathrm{C}$ on usual laboratory media. It lives in moist and dry conditions for a long period of time. It has a free-living capacity and can be found on both animate and inanimate objects. It has been isolated from hospital environments such as foundries and baths, ventilators, contaminated gloves, washcloths, peritoneal dialysate baths, soap dispensers among others. It has also been

${ }^{*}$ Corresponding author. E-mail: ertugrulguclu@hotmail.com. Tel. : (+90) 264 2552100. Fax: (+90) 2642552105.

Abbreviations: MDR, Multidrug resistant; ESBL, extended spectrum beta-lactamase; SWI, sterile water for injection. 
recovered from different human sources, including skin, sputum, urine, faeces, and vaginal secretions (Allen and Hartman, 2005).

Up to $25 \%$ of healthy ambulatory adults exhibit cutaneous colonization and $7 \%$ have transient pharyngeal colonization. It is also the most common gram-negative bacteria persistently carried on the skin of hospital personel. In addition, it has been found that Acinetobacter was the cause of $1 \%$ of all nosocomial blood-stream infections and $3 \%$ of nosocomial pneumonia in United States hospitals. This ratio is 5 and $10 \%$, respectively, for South American Hospitals (Allen et al, 2005).

B-lactamase inhibitors, especially sulbactam, colistin, fluoroquinolones, ceftazidime, carbapenems (imipenem and meropenem), tetracyclines, and tigecycline have been considered to be active against nosocomial $A$. baumannii strains. However, treatment of this microorganism has become a medical challenge because of the emergence of MDR isolates, including resistance to carbapenems, with increasing frequency (Gilad et al, 2008). In a surveillance report (MYSTIC) from worldwide hospitals for the period $2002-2004$, only 76.1 and $74.7 \%$ were susceptible to meropenem and imipenem, respectively. Susceptibility to other antibiotics was also very low; with $51.9 \%$ gentamicin, $40.5 \%$ ciprofloxacin, $39.8 \%$ piperacillin-tazobactam, and $38.1 \%$ ceftazidime (Unal et al, 2005). Of the $A$. baumannii isolates in Turkey, $67 \%$ were MDR with susceptibility rates to meropenem $53 \%$, imipenem $48 \%$, tobramycin $44 \%$, cefepime $37 \%$, ciprofloxacin $29 \%$, piperacillin-tazobactam 26\%, and ceftazidime 22\% (Zarakolu et al., 2006). Moreover, A. baumannii clones resistant to all antibiotics except polymyxin and (more variably) tigecycline are circulating in many countries (Peleg et al., 2008).

It would thus seem essential to develop new therapeutic agents against this growing threat. Today, there are some new antibiotics (dalbavancin, oritavancin among others) directed against gram-positive bacteria (Coates et al., 2011), but there is a lack of production of newer antibiotics (with the exception of doripenem) directed against gram-negative bacteria. Probably returning to the preantibiotic era has become a reality in many parts of the world (Giske et al., 2008).

With a lack of production of new antibiotics, herbal medicine has become popular. Menthae piperitae is one of the most investigated plant sources. It is known that aetheroleum menthae piperitae inhibited the growth in vitro of Staphylococcus aureus, Pseudomonas aeruginosa, Bacillus subtilis, Enterococcus faecalis and Escherichia coli (WHO monographs, 2004a). Also, extracts of folium menthae piperitae have antibacterial activity in vitro. Addition of ground leaves to agar medium inhibited the growth of Salmonella typhimurium, S. aureus and Vibrio parahaemolyticus (WHO monographs, 2004b).

Menthol is a naturally occurring cyclic terpene alcohol of plant origin. Its use is multifold and includes use in oral hygiene products, confectionaries, pharmaceuticals, and cosmetics. It is currently available in both prescribed and over-the-counter medications for various diseases, including gastrointestinal disorders, common cold and respiratory conditions, and musculoskeletal pain (Landau et al., 2012). Its antimicrobial effect may result, from a perturbation of the lipid fraction of the microorganism plasma membrane, resulting in alterations of membrane permeability and in leakage of intracellular materials (Trombetta et al., 25).

Up to the present day, the antimicrobial activity of folium menthae piperitae and menthol against $A$. baumannii has not been investigated. To our knowledge, this article is the first study investigating the efficacy of folium menthae piperitae extract and menthol both alone and in a mixture (2.6\% folium menthae piperitae extract and $6 \%$ menthol dissolved in methanol) for synergistic activity.

\section{MATERIALS AND METHODS}

\section{Plant material and Menthol}

The genus Menthae piperitae $L$. mint leaves $(30 \mathrm{~g})$ were dried at room temperature and processed in methanol $(250 \mathrm{ml})$ with a waring blender for $10 \mathrm{~min}$. The resulting mixture was filtered after two days. A concentration of $26 \mathrm{~g} / \mathrm{L}$ were obtained from the extract. $99 \%$ purity menthol samples were obtained from Sigma-Aldrich. As a mixture, $2.6 \%$ folium menthae piperitae extract and $6 \%$ menthol were dissolved in methanol and mixed.

\section{Strains and growth conditions}

A. baumannii strains were obtained from our Infectious Diseases and Clinical Microbiology laboratory stocks. These stock strains were isolated from various clinics' hospitalized patient clinical specimens, such as urine, sputum, and pus, which were sent for laboratory identification. The antibiotic susceptibility profiles of all isolates were assessed by Kirby Bauer's disc diffusion method according to the recommendations of the Clinical and Laboratory Standards Institute (CLSI) (CLSI, 2008).

The isolates were stored at $-20^{\circ} \mathrm{C}$ in skimmed milk agar until used in this study. Prior to the study, each strain was subcultured on $5 \%$ blood agar at $37^{\circ} \mathrm{C}$ for two consecutive days. From A. baumannii strains obtained in the second passage, bacterial suspensions were prepared in tryptone soya broth (TSB) (Oxoid, Basingstoke, UK), and adjusted to a turbidity equal to McFarland $0.5\left(1.5 \times 10^{8} \mathrm{cfu} / \mathrm{ml}\right)$ (DIN EN 1040, 2005). All strains were studied with both a quantative suspension test and an agar well diffusion method.

When investigating the true bactericidal activity of disinfectant chemicals or antiseptics, their total neutralization is critical. Disinfectant residues can result in a false-negative (no-growth) test. In this study, 3\% Tween 80 (Polyoxyethylene Sorbitan Monooleate, ICN Biomedicals Inc.) was used as a neutralizing agent in accordance with the Deutsches Institut für Normung e.V. standards (DIN EN 1040, 2005).

\section{Quantitative suspension test}

Bactericidal efficacy was determined, strictly following DIN EN 1040 (DIN EN 1040, 2005). Briefly, $1 \mathrm{~mL}$ of the test organism suspension and $1 \mathrm{~mL}$ of sterile water for injection (SWI) were mixed and incubated at room temperature for a contact time of $2 \mathrm{~min}$. afterwards, 8 $\mathrm{mL}$ of the test substance was added. The resulting solutions were incubated at room temperature for a contact time of $1,5,10,30$, and $60 \mathrm{~min}$. At the end of the contact time, $1 \mathrm{~mL}$ of the test solution 
was transferred to $8 \mathrm{~mL}$ of the neutralizing solution and $1 \mathrm{~mL}$ of SWI and neutralized for $5 \mathrm{~min}$. Thereafter, $1 \mathrm{~mL}$ of the neutralized test solution was spread on $5 \%$ blood agar plates and incubated at $37^{\circ} \mathrm{C}$ for 18 to $24 \mathrm{~h}$. After the incubation time, colonies were counted and expressed as colony-forming units per milliliter (cfu/ml). As a negative control, $8 \mathrm{~mL}$ of SWI was used instead of plant extract or menthol and all steps were done simultaneously with the primer test.

\section{Reduction rate calculation in quantitative suspension test}

The reduction rate was calculated as the expression of the microbicidal efficiency, according to the following formula:

$\log _{10}$ reduction $(R)=\log _{10}$ pre-disinfection count $-\log _{10}$ disinfection count

$R>5$ was considered as significant microbicidal efficiency.

\section{Agar well diffusion method}

Each Mueller-Hinton agar plate was inoculated with the microorganism by streaking the swab over the entire sterile agar surface. This procedure was repeated by streaking 2 more times, rotating the plate approximately $60^{\circ}$ each time to ensure even distribution of the inoculum. As a final step, the rim of the agar was also swabbed. Once the agar was solidified, it was punched with eight millimeter diameter wells and filled with $50 \mu \mathrm{L}$ of the test material (menthol, extract of folium menthae piperitae and mixture of menthol + folium mentha piperita extract). The concentration of the plant extracts employed was $26 \mu \mathrm{g} / \mathrm{ml}$. The plaques were incubated at $37^{\circ} \mathrm{C}$ for 18 to $24 \mathrm{~h}$. Subsequently, the plates were examined for bacterial growth inhibition and the inhibition zone diameter (IZD) was measured to the nearest millimeter.

\section{RESULTS}

A total of 20 MDR $A$. baumannii strains that were identified in the Infectious Diseases and Clinical Microbiology laboratory were selected for the study. Their antibiotic resistance profiles are shown in Table 1 . We used methanol for the extraction of the folium of mentha piperita plant. After extraction, a mixture of $2.6 \%$ folium menthae piperitae extract and 6\% menthol dissolved in methanol was prepared. The antimicrobial activity of menthol, folium menthae piperitae and the mixture was assessed using both the quantitative suspension test and the agar well diffusion method.

In the quantitative suspension test method, microbicidal activity was detected with whole test materials (menthol, folium menthae piperitae extract, and the mixture) at all contact times, except folium menthae piperitae extract at a contact time of $1 \mathrm{~min}$. While $\mathrm{R}$ was $<5$ for menthae piperitae extract at $1 \mathrm{~min}$, it was $>5$ for the other test materials at every contact time and for menthae piperitae extract at 5, 10, 30, and 60 min.

In the agar well diffusion method, the antimicrobial activity of test solutions was assessed by measuring IZD and is tabulated in Table 2. Synergy and good antibacterial activity was found between menthol and menthae piperitae leaf extract. While the IZD was $\geq$ $10 \mathrm{~mm}$ for menthol, except for four test bacteria, it was found to be $\geq 10 \mathrm{~mm}$ for 19 (95\%) bacteria with the mixture. Also, the IZD with the mixture was increased for $13(65 \%)$ test bacteria, compared to the IZD with menthol. On the other hand, the antibacterial activity of folium menthae piperitae against $A$. baumannii was found to be inadequate. We found measurable IZD $(8,15,12$ and $20 \mathrm{~mm}$ ) for only four test bacteria with folium menthae piperitae. There was no IZD for the other bacteria (Table 2).

\section{DISCUSSION}

In this study, a mixture of menthol + folium menthae piperitae showed good in vitro antibacterial activity against MDR $A$. baumannii isolates. The $\mathrm{R}$ factor was $>5$ for all contact times in a quantitative suspension test method and the IZD was $\geq 20 \mathrm{~mm}$ for 17 (85\%) $A$. baumannii isolates with an agar well diffusion method. The lowest IZD was $12 \mathrm{~mm}$ with the agar well diffusion method with this compound.

Hospital acquired infections due to $A$. baumannii can increase morbidity, mortality, and length of stay in the hospital as well as hospital costs. The most important problem in the treatment of $A$. baumannii infections is resistance to most available antibiotics (Maragakis et al., 2008). Patients that acquire artificial devices, such as catheters, sutures, and ventilators and those who have unplanned surgery or antimicrobial therapy (carbapenems, third generation cephalosporin, flouroqunilones, aminoglycosides, and metronidazole) within the previous 90 days are at risk of developing $A$. baumannii infections (Howard et al., 2012). A very small number of antibiotics can be used for antibiotic-resistant bacteria such as our A. baumannii strains. Moreover, the production of new antibiotics is very low (Giske et al., 2008). In recent years, the emergence of strains resistant to antibiotics and the lesser side effects of drugs of natural origin compared to synthetic drugs, has led scientists to further studies of drugs of natural origin (Lysakowska et al, 2011).

Previously, the effectiveness of menthae piperitae and menthol against some gram negative and gram positive bacteria was independently investigated. Aridogan et al. (2002) found antibacterial activity against $S$. aureus with mentha piperita. In another study, the authors declared that menthae piperitae essential oil has antibacterial activity against $E$. coli, a multiresistant strain of Shigella sonnei, and Micrococcus flavus. Also, they found fungistatic and fungicidal activity of this oil against Trichophyton tonsurans and Candida albicans (Mimica-Dukic et al., 2003). In our study, the antimicrobial activity of folium menthae piperitae for $A$. baumannii strains was very low. We found measurable IZD in four strains. Their IZD was $8,12,15$ and $20 \mathrm{~mm}$. For the other strains, there was no 
Table 1. Antibiotic susceptibility of $A$. baumannii strains.

\begin{tabular}{cccccccccc}
\hline A. baumannii strain & AK & CAZ & CIP & IMP & SXT & CN & TZP & CRO & SAM \\
\hline 1 & $\mathrm{R}$ & $\mathrm{R}$ & $\mathrm{R}$ & $\mathrm{R}$ & $\mathrm{R}$ & $\mathrm{R}$ & $\mathrm{R}$ & $\mathrm{R}$ & $\mathrm{R}$ \\
2 & $\mathrm{R}$ & $\mathrm{R}$ & $\mathrm{R}$ & $\mathrm{R}$ & $\mathrm{R}$ & $\mathrm{R}$ & $\mathrm{R}$ & $\mathrm{R}$ & $\mathrm{R}$ \\
3 & $\mathrm{R}$ & $\mathrm{R}$ & $\mathrm{R}$ & $\mathrm{R}$ & $\mathrm{R}$ & $\mathrm{R}$ & $\mathrm{R}$ & $\mathrm{R}$ & $\mathrm{R}$ \\
4 & $\mathrm{R}$ & $\mathrm{R}$ & $\mathrm{R}$ & $\mathrm{R}$ & $\mathrm{R}$ & $\mathrm{S}$ & $\mathrm{R}$ & $\mathrm{R}$ & $\mathrm{R}$ \\
5 & $\mathrm{R}$ & $\mathrm{R}$ & $\mathrm{R}$ & $\mathrm{R}$ & $\mathrm{R}$ & $\mathrm{R}$ & $\mathrm{R}$ & $\mathrm{R}$ & $\mathrm{R}$ \\
6 & $\mathrm{R}$ & $\mathrm{R}$ & $\mathrm{R}$ & $\mathrm{R}$ & $\mathrm{R}$ & $\mathrm{R}$ & $\mathrm{R}$ & $\mathrm{R}$ & $\mathrm{R}$ \\
7 & $\mathrm{R}$ & $\mathrm{R}$ & $\mathrm{R}$ & $\mathrm{R}$ & $\mathrm{S}$ & $\mathrm{R}$ & $\mathrm{R}$ & $\mathrm{R}$ & $\mathrm{R}$ \\
8 & $\mathrm{R}$ & $\mathrm{R}$ & $\mathrm{R}$ & $\mathrm{R}$ & $\mathrm{R}$ & $\mathrm{R}$ & $\mathrm{R}$ & $\mathrm{R}$ & $\mathrm{R}$ \\
9 & $\mathrm{R}$ & $\mathrm{R}$ & $\mathrm{R}$ & $\mathrm{R}$ & $\mathrm{R}$ & $\mathrm{R}$ & $\mathrm{R}$ & $\mathrm{R}$ & $\mathrm{R}$ \\
10 & $\mathrm{R}$ & $\mathrm{R}$ & $\mathrm{R}$ & $\mathrm{R}$ & $\mathrm{R}$ & $\mathrm{R}$ & $\mathrm{R}$ & $\mathrm{R}$ & $\mathrm{R}$ \\
11 & $\mathrm{R}$ & $\mathrm{R}$ & $\mathrm{R}$ & $\mathrm{R}$ & $\mathrm{R}$ & $\mathrm{R}$ & $\mathrm{R}$ & $\mathrm{R}$ & $\mathrm{R}$ \\
12 & $\mathrm{R}$ & $\mathrm{R}$ & $\mathrm{R}$ & $\mathrm{R}$ & $\mathrm{R}$ & $\mathrm{R}$ & $\mathrm{R}$ & $\mathrm{R}$ & $\mathrm{R}$ \\
13 & $\mathrm{R}$ & $\mathrm{R}$ & $\mathrm{R}$ & $\mathrm{R}$ & $\mathrm{R}$ & $\mathrm{R}$ & $\mathrm{R}$ & $\mathrm{R}$ & $\mathrm{R}$ \\
14 & $\mathrm{R}$ & $\mathrm{R}$ & $\mathrm{R}$ & $\mathrm{R}$ & $\mathrm{R}$ & $\mathrm{R}$ & $\mathrm{R}$ & $\mathrm{R}$ & $\mathrm{R}$ \\
15 & $\mathrm{R}$ & $\mathrm{R}$ & $\mathrm{R}$ & $\mathrm{R}$ & $\mathrm{R}$ & $\mathrm{S}$ & $\mathrm{R}$ & $\mathrm{R}$ & $\mathrm{R}$ \\
16 & $\mathrm{R}$ & $\mathrm{R}$ & $\mathrm{R}$ & $\mathrm{R}$ & $\mathrm{R}$ & $\mathrm{R}$ & $\mathrm{R}$ & $\mathrm{R}$ & $\mathrm{R}$ \\
17 & $\mathrm{R}$ & $\mathrm{R}$ & $\mathrm{R}$ & $\mathrm{R}$ & $\mathrm{R}$ & $\mathrm{R}$ & $\mathrm{R}$ & $\mathrm{R}$ & $\mathrm{R}$ \\
18 & $\mathrm{R}$ & $\mathrm{R}$ & $\mathrm{R}$ & $\mathrm{R}$ & $\mathrm{R}$ & $\mathrm{R}$ & $\mathrm{R}$ & $\mathrm{R}$ & $\mathrm{R}$ \\
19 & $\mathrm{R}$ & $\mathrm{R}$ & $\mathrm{R}$ & $\mathrm{R}$ & $\mathrm{R}$ & $\mathrm{R}$ & $\mathrm{R}$ & $\mathrm{R}$ & $\mathrm{R}$ \\
20 & $\mathrm{R}$ & $\mathrm{R}$ & $\mathrm{R}$ & $\mathrm{R}$ & $\mathrm{R}$ & $\mathrm{R}$ & $\mathrm{R}$ & $\mathrm{R}$ & $\mathrm{R}$ \\
\hline
\end{tabular}

AK, Amikacin; CAZ, ceftazidime; CIP, ciprofloxacin; IMP, imipenem; SXT, trimethoprim-sulfamethazole; CN, gentamicin; TZP, piperacillin-tazobactam; CRO, ceftriaxone; SAM, ampicillin-sulbactam.

Table 2. Inhibition zone diameters (in millimeters) of menthol, Menthae Piperitae and the mixture against $A$. baumannii.

\begin{tabular}{lccc}
\hline A. baumannii strain & $\begin{array}{c}\text { Mixture of menthol }+ \text { folium } \\
\text { menthae piperitae }(\mathbf{m m})\end{array}$ & $\begin{array}{c}\text { Menthol } \\
(\mathbf{m m})\end{array}$ & $\begin{array}{c}\text { Folium menthae } \\
\text { piperitae extract }(\mathbf{m m})\end{array}$ \\
\hline Strain 1 & 12 & 0 & 0 \\
Strain 2 & 25 & 20 & 0 \\
Strain 3 & 20 & 12 & 8 \\
Strain 4 & 20 & 12 & 0 \\
Strain 5 & 25 & 20 & 0 \\
Strain 6 & 20 & 8 & 0 \\
Strain 7 & 30 & 30 & 17 \\
Strain 8 & 40 & 10 & 0 \\
Strain 9 & 20 & 10 & 0 \\
Strain 10 & 25 & 25 & 20 \\
Strain 11 & 20 & 15 & 0 \\
Strain 12 & 20 & 10 & 0 \\
Strain 13 & 15 & 0 & 0 \\
Strain 14 & 20 & 15 & 0 \\
Strain 15 & 15 & 20 & 0 \\
Strain 16 & 35 & 30 & 0 \\
Strain 17 & 25 & 0 \\
Strain 18 & 25 & 30 & 0 \\
Strain 19 & 30 & 30 & 0 \\
Strain 20 & 25 & 35 & 12 \\
\hline
\end{tabular}

inhibition zone.

In a quantitative suspension test, the $\mathrm{R}$ factor was found to be $<5$ with a contact time of $1 \mathrm{~min}$, and $>5$ at the other contact times $(5,10,30$, and $60 \mathrm{~min})$ for the menthae 
piperitae extract. These findings suggest a time-dependent effect of the menthae piperitae extract against $A$. baumannii. In our opinion, this effect should be studied with more $A$. baumannii isolates and with other Gram positive and negative bacteria. In addition, menthol has antibacterial activity against a variety of microorganisms, including both Gram positive and Gram negative bacteria (Patel et al., 2007). Schelz et al. (2006) has found efficacy against Staphylococcus epidermidis and E. coli. In another study, Trombetta et al. (2005) found strong antibacterial activity against $S$. aureus. Our results indicate that menthol has significant antibacterial activity against A. baumannii. The IZD was measured as $\geq 20 \mathrm{~mm}$ for 10 $(50 \%)$ strains and $\geq 10 \mathrm{~mm}$ for $17(85 \%)$ strains. Also, the $\mathrm{R}$ factor was $>5$ for menthol at every contact time.

The results shown in Table 2 indicate that the combination of menthol and folium menthae piperitae possessed better antimicrobial activity and showed a synergy between the two substances. In $13(65 \%)$ A. baumannii strains, the IZD was larger with the mixture of menthol + menthae piperitae. In our opinion, this situation is due to the menthol, which is the main component of menthae piperitae, since the major constituent of the leaves is the essential oil $(0.5-4 \%)$ and $30-55 \%$ of this oil is menthol (WHO monographs, 2004a). Our results confirm this theory as well. While the $R$ factor was $<5$ at the $1 \mathrm{~min}$ contact time for the menthae piperitae extract, it was $>5$ at the other contact times.

In conclusion, menthol shows antibacterial activity against A. baumannii in vitro. Its topical form can be used in skin and soft tissue infections, burn wound infections, diabetic foot infections and decubitus wound infections due to $A$. baumannii. Further studies focused on the safety of topical application and its dermal direct absorption should be carried out. In addition, the effectiveness of menthol against problem bacteria such as MDR Pseudomonas aeruginosa, ESBL producing Enterobacteriaceae and Klebsiella spp. should be investigated.

\section{ACKNOWLEDGMENTS}

This project was supported by Sakarya University BAP. BAP project no: 2012-02-04-031.

\section{REFERENCES}

Allen DM and Hartman BJ (2005). Acinetobacter species. In: Mandell GL, Bennett JE, Dolin R, eds. Principles and practice of infectious diseases. 6th ed. Philadelphia: Elsevier Churchill Livingstone; 263236.

Aridogan BC, Baydar H, Kaya S, Demirci M, Ozbasar D, Mumcu E (2002). Antimicrobial activity and chemical composition of some essential oils. Arch. Pharm Res. 25(6):860-4

CDC, Centers for Disease Control and Prevention (2010). Acinetobacter in Healthcare Settings. Available at: <http://www.cdc.gov/HAl/organisms/acinetobacter.html> Accessed February 152012.

CLSI, Clinical and Laboratory Standards Institute (2008). Performance Standarts for Antimicrobial Susceptibility Testing; Eighteenth Informational Supplement. CLSI document M100-S18. Wayne, PA: Clinical and Laboratory Standarts Institute.
Coates AR, Halls G, Hu Y (2011). Novel classes of antibiotics or more of the same? Br J Pharmacol. 163(1):184-94.

Deutsches Institut für Normung. (2005). DIN EN 1040: Chemical Disinfectants and Antiseptics-Quantitative Suspension Test for the Evaluation of BasicBactericidal Activity of Chemical Disinfectants and Antiseptics-Test Method and Requirements (Phase 1). Berlin, Germany: Beuth.

Gilad J and Carmeli Y (2008). Treatment options for multidrug-resistant Acinetobacter species. Drugs. 68(2):165-89.

Giske CG, Monnet DL, Cars O, Carmeli Y. (2008). ReAct-Action on Antibiotic Resistance. Clinical and economic impact of common multidrug-resistant gram-negative bacilli. Antimicrob. Agents Chemother. 52(3):813-21.

Howard A, O'Donoghue M, Feeney A, Sleator RD (2012). Acinetobacter baumannii: An emerging opportunistic pathogen. Virulence. 3(3):243250

Kunz AN and Brook I (2010). Emerging resistant Gram-negative aerobic bacilli in hospital-acquired infections. Chemotherapy. 56(6):492-500.

Landau E and Shapira R (2012). Effects of subinhibitory concentrations of menthol on adaptation, morphological, and gene expression changes in enterohemorrhagic Escherichia coli. Appl. Environ. Microbiol. 78(15):5361-7.

Lysakowska M, Denys A, Sienkiewicz M (2011). The activity of thyme essential oil against Acinetobacter spp. Cent. Eur. J. Biol. 6(3):405413.

Maragakis LL and Perl TM (2008). Acinetobacter baumannii: epidemiology, antimicrobial resistance, and treatment options. Clin Infect Dis. 46(8):1254-63.

Mimica-Dukic N, Bozin B, Sokovic M, Mihajlovic B, Matavulj M. (2003). Antimicrobial and antioxidant activities of three Mentha species essential oils. Planta Med. 69(5):413-9.

Patel T, Ishiuji Y, Yosipovitch G (2007). Menthol: a refreshing look at this ancient compound. J Am Acad Dermatol. 57(5):873-8.

Peleg AY, Seifert H, Paterson DL (2008). Acinetobacter baumannii: emergence of a successful pathogen. Clin Microbiol Rev. 21(3):53882.

Rosenthal VD, Maki DG, Jamulitrat S, Medeiros EA, Todi SK, Gomez DY, Leblebicioglu H, Abu Khader I, Miranda Novales MG, Berba R, Ramírez Wong FM, Barkat A, Pino OR, Duenas L, Mitrev Z, Bijie H, Gurskis V, Kanj SS, Mapp T, Hidalgo RF, Ben Jaballah N, Raka L, Gikas A, Ahmed A, Thu le TA, Guzman Siritt ME, INICC Members (2010). International Nosocomial Infection Control Consortium (INICC) report, data summary for 2003-2008, issued June 2009. Am. J. Infect. Control. 38: 95-104.

Schelz Z, Molnar J, Hohmann J (2006). Antimicrobial and antiplasmid activities of essential oils. Fitoterapia. 77(4):279-85.

Trombetta D, Castelli F, Sarpietro MG, Venuti V, Cristani M, Daniele C, Saija A, Mazzanti G, Bisignano G (2005). Mechanisms of antibacterial action of three monoterpenes. Antimicrob Agents Chemother. 49(6):2474-8.

Unal S, Garcia-Rodriguez JA (2005). Activity of meropenem and comparators against Pseudomonas aeruginosa and Acinetobacter spp. isolated in the MYSTIC Program, 2002-2004. Diagn Microbiol Infect Dis. 53(4):265-71.

WHO, World Health Organization Monographs. (2004a) Aetheroleum Menthae Piperitae. WHO Monographs on selected medicinal plants. P: 188-198 Available at: <http://apps.who.int/medicinedocs/pdf/s4927e/s4927e.pdf> Accessed January 182012.

WHO, World Health Organization Monographs (2004b). Folium Menthae Piperitae. WHO Monographs on selected medicinal plants. P: $\quad 199-205 . \quad$ Available at: <http://apps.who.int/medicinedocs/pdf/s4927e/s4927e.pdf> Accessed January 182012.

Zarakolu P, Hascelik G, Unal S (2006). Antimicrobial susceptibility pattern of nosocomial gram negative pathogens: results from MYSTIC study in Hacettepe University Adult Hospital (2000-2004). Mikrobiyol Bul. 40(3):147-54 